\title{
Ultraviolet irradiation represses TGF- $\beta$ type II receptor transcription through a 38-bp sequence in the proximal promoter in human skin fibroblasts
}

\author{
Tianyuan He, Taihao Quan and Gary J. Fisher
}

Department of Dermatology, University of Michigan, Ann Arbor, MI, USA

Correspondence: Gary J. Fisher, PhD, University of Michigan - Dermatology, 1301 E Catherine Street, 6447 Med Sci I, Ann Arbor, MI 48109-5609, USA, Tel.: 734-763-1469, Fax: 734-647-0076, e-mail: dianemch@umich.edu

Abstract: Transforming growth factor- $\beta$ (TGF- $\beta$ ) is a major regulator of collagen gene expression in human skin fibroblasts. Cellular responses to TGF- $\beta$ are mediated primarily through its cell surface type I (T $\beta R I)$ and type II (T $\beta$ RII) receptors.

Ultraviolet (UV) irradiation impairs TGF- $\beta$ signalling largely due to reduced T $\beta$ RII gene expression, thereby decreasing type I procollagen synthesis, in human skin fibroblasts. UV irradiation does not alter either T $\beta$ RII mRNA or protein stability, indicating that UV reduction in T $\beta$ RII expression likely results from transcriptional or translational repression. To understand how UV irradiation regulates T $\beta$ RII transcription, we used a series of T $\beta$ RII promoter-luciferase 5 -deletion constructs (covering $2 \mathrm{~kb}$ of the TRRII proximal promoter) to determine transcriptional rate in response to UV irradiation. We identified a 137-bp region upstream of the transcriptional start site that exhibited high promoter activity and was repressed $60 \%$ by UV irradiation, whereas all other T $\beta$ RII promoter reporter constructs exhibited either low promoter activities or no regulation by UV irradiation. Mutation of potential transcription factor binding sites within the promoter region revealed that an inverted
CCAAT box ( -81 bp from transcription start site) is required for promoter activity. Mutation of the CCAAT box completely abolished UV irradiation regulation of the T $\beta$ RII promoter. Protein-binding assay, as determined by electrophoretic mobilityshift assays (EMSAs) using the inverted CCAAT box as probe $(-100 /-62)$, demonstrated significantly enhanced protein binding in response to UV irradiation. Super shift experiments indicated that nuclear factor $\mathrm{Y}$ (NFY) is able to binding to this sequence, but NFY binding was not altered in response to UV irradiation, indicating additional protein(s) are capable of binding this sequence in response to UV irradiation. Taken together, these data indicate that UV irradiation reduces T $\beta$ RII expression, at least partially, through transcriptional repression. This repression is mediated by a 38 -bp sequence in T $\beta$ RII promoter, in human skin fibroblasts.

Key words: TGF- $B$ - transcription - ultraviolet

Accepted for publication 24 March 2014

\section{Introduction}

The most abundant structural protein in human skin is type I procollagen, which is responsible for the skin's strength and resiliency. Dermal fibroblasts are primary cellular source for type I collagen synthesis. Alteration of skin collagen content and organization impair wound healing, contribute to skin cancer and are responsible for skin fragility in the elderly (1-4).

Transforming growth factor- $\beta$ (TGF- $\beta$ ) is a major regulator of collagen gene expression in human skin fibroblasts. Cellular responses to TGF- $\beta$ are mediated primarily through its cell surface type I receptor (T $\beta R I$ ) and type II receptor (T $\beta R I I)$. Binding of TGF- $\beta$ to T $\beta$ RII activates the intrinsic serine/threonine kinase activity of T $\beta$ RI, which phosphorylates transcription factors Smad2 and Smad3. Phosphorylated Smad2 and Smad3 combine with Smad4 and translocate into the nucleus, where they function to regulate transcription of specific genes that possess TGF- $\beta$ response elements in their promoters (5-7).

Ultraviolet (UV) irradiation from the sun is a potent environmental hazard capable of damaging cellular DNA and causing mutations $(8,9)$. In addition, solar UV irradiation is the primary cause of premature skin ageing (photoageing). We have reported previously that UV irradiation reduces type I procollagen production through impairment of TGF- $\beta$ signal transduction pathway. This reduction is largely due to repression of T $\beta$ RII gene expression $(10,11)$. We observed that UV irradiation significantly represses T $\beta R I I$ mRNA and protein, but not T $\beta$ RI, in cultured human skin fibroblasts. In the same study, we also observed that neither T $\beta$ RII mRNA stability nor T $\beta$ RII protein stability was altered after UV exposure, indicating that UV repression of T $\beta$ RII mRNA and protein must result from reduced mRNA transcription or protein synthesis.

We report here that UV irradiation reduces T $\beta$ RII expression, at least partially, through transcriptional repression. This repression is mediated by a 38 -bp sequence in T $\beta$ RII promoter, in human skin fibroblasts.

\section{Materials and methods}

Materials

Dulbecco's modified Eagle's medium, foetal calf serum, trypsin solution, penicillin/streptomycin and L-glutamine were purchased from Gibco (Invitrogen, Carlsbad, CA, USA). $\left[\gamma_{-}{ }^{32} \mathrm{p}\right]$ ATP was obtained from Perkin Elmer (Boston, MA, USA). Type I procollagen, T $\beta$ RII and NF-Y antibodies were purchased from Santa Cruz Biotechnology (Santa Cruz, CA, USA). All other reagents were purchased from Sigma-Aldrich Company (St. Louis, MO, USA). 


\section{Cell culture and UV irradiation}

Adult human skin primary dermal fibroblasts were isolated from punch biopsy of healthy adult normal human skin, as described previously (10). Cells were cultured at $37^{\circ} \mathrm{C}$ under $5 \% \mathrm{CO}_{2}$ in Dulbecco's modified Eagle's medium supplemented with $10 \%$ foetal bovine serum. Cells were used between passages 5 and 10. UV irradiation of human skin primary dermal fibroblasts was performed as described previously (10-12). Briefly, subconfluent cells were irradiated with a UVB/A2 source $\left(50 \mathrm{~mJ} / \mathrm{cm}^{2}\right)$ using an Ultralite Panelite lamp containing six FS24T12 UVB-HO bulbs. The UV irradiation intensity was monitored with an IL1400A phototherapy radiometer and a SED240/UVB/W photodetector (International Light, Newbury, MA, USA). A Kodacel filter was used to remove UVC (wavelengths below $290 \mathrm{~nm}$ ).

Promoter/reporter constructs

A series of T $\beta$ RII promoter-luciferase $5^{\prime}$-deletion constructs (covering $2 \mathrm{~kb}$ of T $\beta$ RII proximal promoter) was kindly provided by Dr. Seong-Jin Kim (National Institute of Health, Bethesda, MD, USA) (13). Luciferase reporters driven by wild-type and mutant T $\beta$ RII promoter spanning from -137 to -47 were generated by PCR using mutant templates, which were kindly provided by Dr. James W. Freeman (Department of Medicine, University of Texas Health Service Center, San Antonio, TX, USA) (14). PCR primers were forward primer 5'-CGCTCGAGTGAGGGGCAGCTGAAAGTC-3'; reverse primer to generate wild-type and mutants (Sp1A, Sp1C and inverted CCAAT) reporters was $5^{\prime}$-GCAAGCTTACGTCCAGCCCC TAG-3'; reverse primer for Sp1D mutant was $5^{\prime}$-GCAAGCTT-AC GTCGAATTCCTAG-3'; wild-type and mutant T $\beta$ RII promoterluciferase reporters, spanning from -100 to -62 , were generated by PCR using wild-type and mutant templates described above. PCR primers were as follows: forward primer: $5^{\prime}$-CGCTCGAGGGC-T GGTCTAGGAAAC-3' and reverse primer: $5^{\prime}$-GCCTCGAGCAGCTACGAGAGAGC-3'. The PCR products were cloned into pGL-3 luciferase reporter using Hind III and Xho I restriction sites.

Transient transfection of T $\beta$ RII promoter constructs and luciferase assays

Human skin fibroblasts were transiently transfected and cotransfected with a $\beta$-galactosidase, to provide an internal control for transfection efficiency, and the luciferase reporters described above. Transfection was performed by electroporation, according to the manufacturer's protocol (Amaxa Biosystems, Gaithersburg, MD, USA). Aliquots containing identical $\beta$-galactosidase activity were used for each luciferase assay. Luciferase activity was measured using an enhanced luciferase assay kit (PharMingen International, San Diego, CA, USA) according to the manufacturer's protocol.

Electrophoretic mobility-shift assay and super shift assay Electrophoretic mobility-shift assays (EMSAs) were performed as described previously $(10,15)$. Nuclear extracts were prepared by nuclear and cytoplasmic extraction reagents (Pierce, Rockford, IL, USA). Double-stranded oligonucleotide probes for EMSAs were as follows: wild-type T $\beta$ RII promoter probe spanning -100 to -62 (38 bp), 5'-GGCTGGTCTAGGAAACATGATTGGCA-GCTACGAG AGAG- $3^{\prime}$ and mutant T $\beta$ RII promoter probe, $5^{\prime}$-GGCTGGTCTAGGAAACATGGTGTACAGCTACGAGAGAG-3'. Consensus wildtype and mutant nuclear factor Y (NFY) probes were purchased from Santa Cruz Biotechnology. All other oligonucleotides were synthesized by Invitrogen. For competition experiments, a 10- to 50-fold molar excess of non-radioactive competitor probes was preincubated with the nuclear extract for $30 \mathrm{~min}$ on ice before $\left[{ }^{32} \mathrm{P}\right]$ probes were added. For antibody super shift assays, reactions were performed by pre-incubating nuclear extracts $(20 \mu \mathrm{g})$ with antibody $(2 \mu \mathrm{g})$ on ice for $30 \mathrm{~min}$ and then incubated with $\left[{ }^{32} \mathrm{P}\right]$ probes. Gels were transferred to $3 \mathrm{MM}$ Whatman paper, vacuum-dried and scanned using the STORM MolecularImager (Molecular Dynamics).

\section{RNA isolation and quantitative real-time RT-PCR}

Total RNA was isolated using TRIzol reagent (Invitrogen, Carlsbad, CA, USA) according to the manufacturer's protocol. Total RNA (100 ng) was reverse-transcribed using Taqman Reverse Transcription kit (Applied Biosystems, Foster City, CA, USA). Real-time PCR was performed on a 7900 Sequence Detector (Applied Biosystems) using Taqman Universal PCR Master Mix Reagents (Applied Biosystems). PCR primers and probes were purchased from Applied Biosystems. Type I procollagen, T $\beta$ RII and 36B4 primers and probes were described previously (15). Target gene levels were normalized to the housekeeping gene $36 \mathrm{~B} 4$, as an internal control for quantification.

\section{Western blot analysis}

Western analysis was performed as previously described (10). Briefly, whole-cell extracts were prepared from cells using wholecell extraction buffer ( $25 \mathrm{~mm}$ HEPES, $0.3 \mathrm{M} \mathrm{NaCl}, 1.5 \mathrm{~mm} \mathrm{MgCl}_{2}$, $0.2 \mathrm{~mm}$ EDTA, 0.1\% Triton X-100, $0.5 \mathrm{~mm}$ DTT, $20 \mathrm{~mm} \beta$-glycerolphosphate, $0.1 \mathrm{~mm} \mathrm{Na} \mathrm{VO}_{4}, 2 \mu \mathrm{g} / \mathrm{ml}$ leupeptin and $100 \mu \mathrm{g} / \mathrm{ml}$ PMSF). Protein concentrations were measured by Bio-Rad protein assay (Bio-Rad Laboratories, Hercules, CA, USA). Proteins were resolved on $6 \%$ or $10 \%$ SDS-PAGE, transferred to PVDF membrane and reacted with primary antibodies. Protein bands were visualized and quantified with enhanced chemifluorescence (ECF) (Vistra ECF Western Blotting System, GE Healthcare, Piscataway, NJ, USA) following the manufacturer's protocol. The intensities of each band were quantified by STORM MolecularImager (Molecular Dynamics, Sunnyvale, CA, USA) and normalized using $\beta$-actin as loading control.

\section{Transfection and siRNA}

Human skin fibroblasts were transiently transfected with control siRNA (5'-UUCUCCGAACGUGUCACGU-3', Qiagen, Chatsworth, CA), or NFY-A siRNA (5'-CCAUCGUCUAUCAACCAGUUA- $\left.3^{\prime}\right)$, and designed to target exon 6 of NFY-A (16) (Sigma-Aldrich Company, St Louis, MO, USA), as described previously (17). All siRNA were transiently transfected into dermal fibroblasts by electroporation (Amaxa Biosystems, Koeln, Germany) as described above. Forty-eight hours after transfection, whole-cell extract and total RNA were prepared. Protein and mRNA levels were determined by Western blot analysis and quantitative real-time RT-PCR, respectively, as described above.

\section{Statistical analysis}

Comparisons between groups were determined with the Student's $t$-test. All $P$-values are two-tailed and considered significant when $P<0.05$.

\section{Results}

UV irradiation represses T $\beta$ RII promoter activity in human skin fibroblasts

Ultraviolet (UV) irradiation significantly reduces type I procollagen gene expression through impairment of TGF- $\beta$ signal transduction 
pathway, largely due to repression of T $\beta$ RII gene expression (11). This impairment is not due to reduced stability of either TßRII mRNA or protein, indicating that UV repression of expression likely results from reduced synthesis of T $\beta$ RII mRNA or protein (data not shown). To determine whether UV irradiation alters TRRII mRNA transcription, we transiently transfected a series of T $\beta$ RII promoter-luciferase $5^{\prime}$-deletion constructs (covering $2 \mathrm{~kb}$ of the T $\beta$ RII proximal promoter) into cultured skin fibroblasts. Twenty-four hours after transfection, cells were exposed to UV $\left(50 \mathrm{~mJ} / \mathrm{cm}^{2}\right)$. Twenty-four hours after UV irradiation, luciferase activity was determined. These studies identified a 137 base pair region upstream of the transcriptional start site that exhibited high promoter activity and was significantly repressed $60 \%(n=8$, $P<0.05$ ) by UV irradiation (Fig. 1). The reporter construct pT $\beta$ RII-47/+54 also exhibited high promoter activity but was not altered by UV irradiation. These data indicate that the T $\beta R I I$ promoter from -137 to -46 contains transcriptional regulation element(s) that is required for T $\beta$ RII promoter activity and regulated by UV irradiation.

\section{TRRII promoter activity requires inverted CCAAT box in} human skin fibroblasts

Analysis of the T $\beta$ RII promoter from -137 to -47 revealed three potential SP1-binding sites and one inverted CCAAT transcription regulation site. To determine which of these sites are required for promoter activity and confer regulation by UV irradiation, we generated a series of mutant T $\beta$ RII promoter luciferase reporter constructs (covering the -137 to -47 base pair region). Mutation of each SP1 site did not alter either promoter activity or repression by UV irradiation (Fig. 2). In contrast, mutation of the inverted CCAAT box significantly reduced both T $\beta$ RII promoter activity and responsiveness to UV irradiation (Fig. 2). These results indicate that the inverted CCAAT box is critical element for T $\beta$ RII promoter regulation.

To further examine the activity of the inverted CCAAT site, we generated a 38 bp T $\beta$ RII promoter construct $(-100 /-62)$ containing the inverted CCAAT site, but excluding the SP1 sites. After transfection and UV irradiation, we found that this $38 \mathrm{bp}$ region retained full promoter, that is, activity similar to the $-137 /-47$ construct (Fig. 2), and was repressed by UV irradiation (Fig. 3). Mutation of the inverted CCAAT site caused loss of promoter

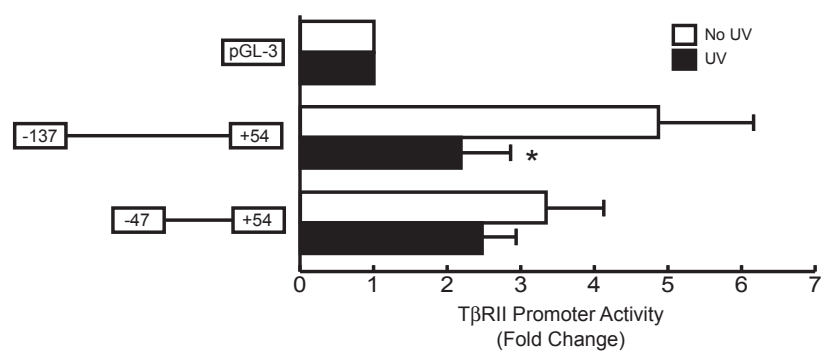

Figure 1. Ultraviolet (UV) irradiation represses T $\beta R \| l$ promoter activity in human skin fibroblasts. Human skin fibroblasts were transiently transfected with T $\beta R$ II promoter (sequence from -137 to +54 and -47 to +54 ) luciferase reporter constructs and $\beta$-galactosidase expression vector. Cells were exposed to UV irradiation $\left(50 \mathrm{~mJ} / \mathrm{cm}^{2}\right) 24 \mathrm{~h}$ after transfection, and cell lysates were prepared $24 \mathrm{~h}$ post-UV irradiation. Luciferase activities were normalized to $\beta$-galactosidase activity Data are means \pm SEM for fold change in luciferase activity relative to activity in cells transfected with control vector, pGL-3 luciferase reporter. $n=8 . * P<0.05$ versus non-UV-irradiated cells.

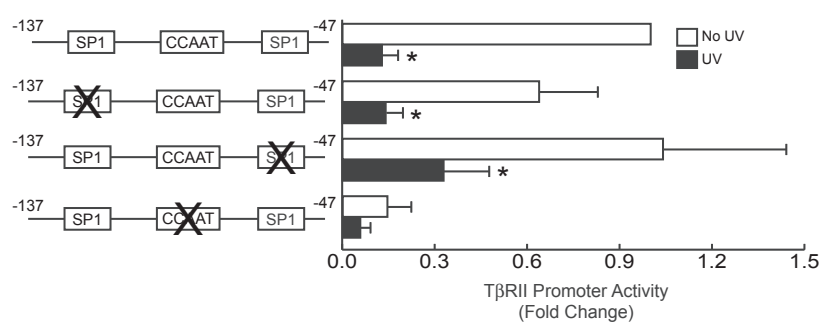

Figure 2. T $\beta R \|$ promoter activity requires inverted CCAAT box in human skin fibroblasts. Human skin fibroblasts were transiently transfected with wild-type TRRII promoter (sequence from -137 to -47 ) luciferase reporter construct, or mutant T $\beta R$ II promoter luciferase constructs in which Sp1 or inverted CCAAT box was mutated. Cells were cotransfected with the $\beta$-galactosidase expression vector. Cells were exposed to ultraviolet (UV) irradiation $\left(50 \mathrm{~mJ} / \mathrm{cm}^{2}\right) 24 \mathrm{~h}$ after transfection, and cell lysates were prepared $24 \mathrm{~h}$ post-UV irradiation. Luciferase activities were normalized to $\beta$-galactosidase activity. Data are means \pm SEM for fold change in luciferase activity relative to activity in cells transfected with wild-type T $\beta R \|$ promoter luciferase construct. $n=6 . * P<0.05$ versus non-UV-irradiated cells.

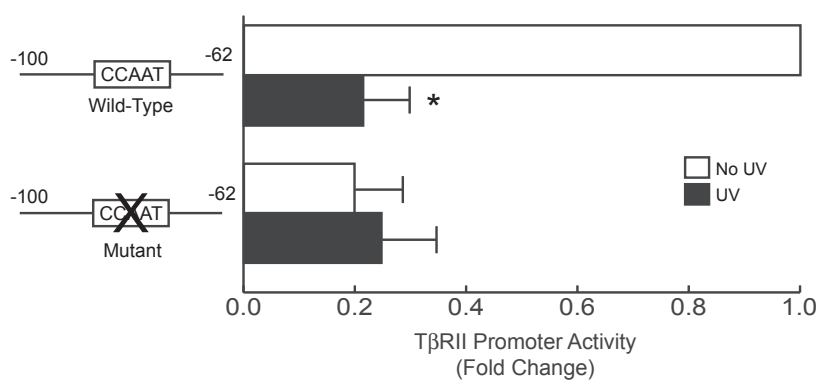

Figure 3. Inverted CCAAT box confers ultraviolet (UV) irradiation repression to TRRII promoter in human skin fibroblasts. Human skin fibroblasts were transiently transfected with the wild-type T $\beta R \| I$ promoter (sequence from -100 to -62 )

luciferase reporter construct, or mutant T $\beta R$ Il promoter luciferase reporter construct in which the inverted CCAAT box was mutated. Cells were co-transfected with the $\beta$-galactosidase expression vector. Cells were exposed to UV irradiation $(50 \mathrm{~mJ} /$ $\mathrm{cm}^{2}$ ) $24 \mathrm{~h}$ after transfection, and cell lysates were prepared $24 \mathrm{~h}$ post-UV irradiation. Luciferase activities were normalized to $\beta$-galactosidase activity. Data are means \pm SE for fold change in luciferase activity relative to activity of wild-type TRRII promoter luciferase reporter construct. $n=4$. $* P<0.05$ versus non-UVirradiated cells.

activity. Taken together, these data indicate that the inverted CCAAT sequence located between -100 to -62 base pairs is required and sufficient for T $\beta$ RII proximal promoter activity and confers responsiveness to UV irradiation.

UV irradiation increases protein binding to inverted CCAAT box in the TßRII proximal promoter in human skin fibroblasts

Ultraviolet (UV) irradiation repression of the T $\beta$ RII promoter may reflect altered protein binding to the inverted CCAAT region of the promoter. To examine this possibility, we performed EMSA using T $\beta$ RII promoter $-100 /-62$ as probe (Fig. 4a). UV irradiation substantially increased the amount of the retarded DNA-protein complex (Fig. 4b). This increased protein binding occurred $2 \mathrm{~h}$ post-UV irradiation and remained increased for at least $8 \mathrm{~h}$ post-UV exposure. No DNA-protein complex was detected with mutant CCAAT probe (Fig. $4 \mathrm{~b}$, right panel). To confirm the specificity of the retarded complexes formed with the wild-type probe, we performed competition EMSA. DNA-protein complex with the labelled $-100 /-62$ probe was completely abolished by excess unlabelled the wild-type probe, but not by the mutant CCAAT probe (Fig. 4c). 
(a) -100 TRRII Wt GGCTGGTCTAGGAAACATGATTGGCAGCTACGAGAGAG TBRII Mut

(b)
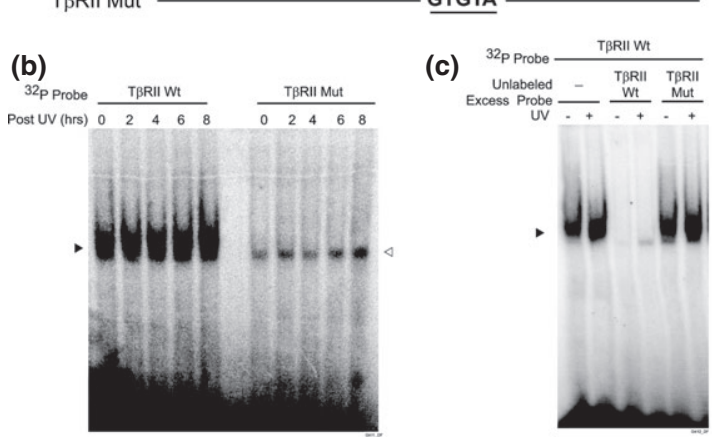

Figure 4. Ultraviolet (UV) irradiation increases protein binding to inverted CCAAT box in the TRRII proximal promoter in human skin fibroblasts. (a) Sequence of TRRII promoter spanning nucleotides -100 to -62 used as probes for electrophoretic mobility-shift assays (EMSAs). Wild-type and mutant-inverted CCAAT sites are bolded and underlined, respectively. (b) EMSAs were performed using [ $\left.{ }^{32} \mathrm{P}\right] \mathrm{T} \beta \mathrm{R} \|$ wild-type or mutant probes. Nuclear extracts were prepared at indicated times after UV irradiation $\left(50 \mathrm{~mJ} / \mathrm{cm}^{2}\right)$. Closed triangle indicates specific retarded complex. Open triangle indicate non-specific bands. (c) Competition of protein binding to TBRII wild-type probe by 50 -fold molar excess unlabelled wild-type or mutant probe. Nuclear extracts were prepared $6 \mathrm{~h}$ after UV irradiation. Closed triangle indicates specific retarded complex. Results are representative of three experiments.

NFY binds to the TßRII promoter activity but binding is not altered by UV in human skin fibroblasts

The CCAAT box is one of the most common cis-elements present in eukaryotic promoters and can serve as a binding site for transcription factor NFY. To determine whether NFY interacts with the inverted CCAAT box in the TRRII promoter, we performed competition EMSA with labelled wild-type T $\beta$ RII promoter $-100 /-62$ probe and excess unlabelled NFY consensus probe. As shown in Fig. 5a, addition of excess NFY consensus probe reduced protein binding to the T $\beta$ RII probe. Similar reduction in binding was observed in samples from both non-irradiated and UV-irradiated dermal fibroblasts. In contrast, competition with excess mutant NFY probe did not alter formation of the retarded complexes, indicating that competition with NFY consensus probe was specific. To further confirm NFY binding, we performed super shift assays with antibody that specifically recognizes the NFY-A subunit of NFY. Super-shifted DNA-protein-antibody complexes were detected in samples from both UV-irradiated and non-UV-irradiated dermal fibroblasts (Fig. 5b). These data indicate that NFY binds to the $-100 /-62$ sequence in the T $\beta$ RII proximal promoter.

We next investigated whether NFY DNA binding is altered by UV irradiation. To examine this question, we performed EMSA and super shift EMSA with $\left[{ }^{32} \mathrm{P}\right]$ NFY consensus probe and antiNFY-A antibody. As shown in Fig. 5c, retarded complexes and super-shifted bands were readily detected in nuclear extracts from non-irradiated skin fibroblasts. Notably, the intensities of both retarded and super-shifted bands were not altered by treatment of fibroblasts with UV irradiation.

Taken together, these data indicate that NFY binds to the $38 \mathrm{bp}$ region of the T $\beta$ RII promoter, and this binding is not regulated by UV irradiation. Therefore, increased NFY DNA binding is not responsible for increased DNA-protein complex formation with the TßRII promoter, which is observed following UV irradiation.

\section{Discussion}

Transcriptional regulation of T $\beta$ RII gene expression plays a key role in modulating TGF- $\beta$ responsiveness. Reduced expression of T $\beta$ RII has been identified in several types of tumor cells. Transfection of wild-type T $\beta$ RII construct into such tumor cells is able to restore their sensitivity to TGF- $\beta$ and suppress cell growth $(18,19)$. (a)

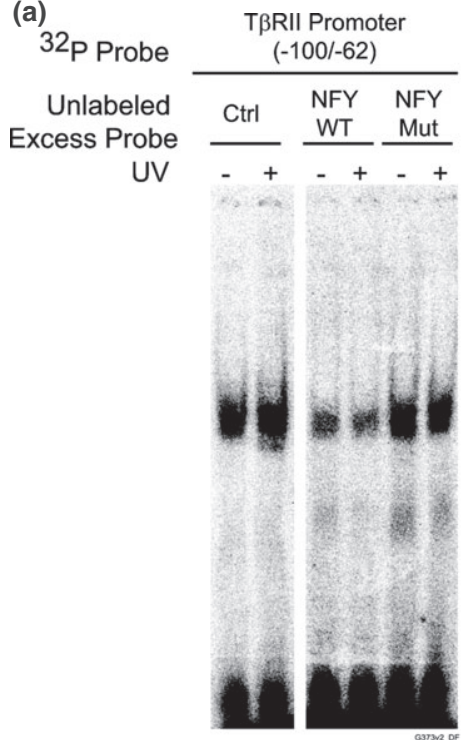

(b)

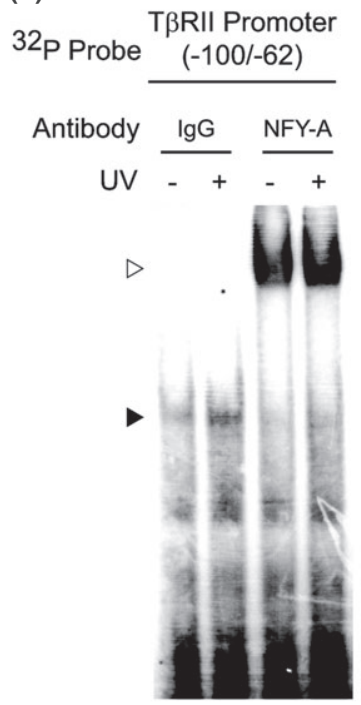

(c)

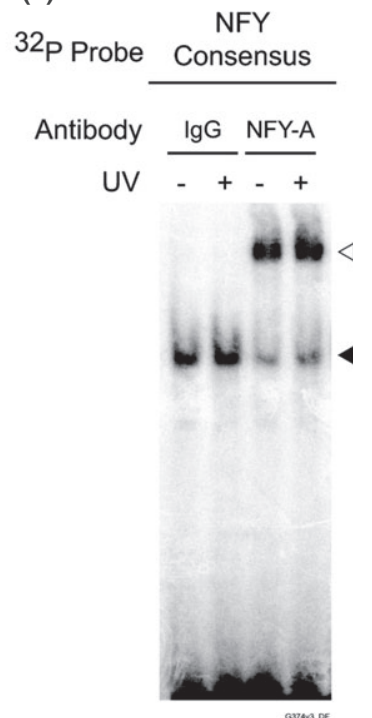

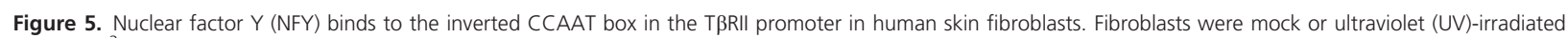
$\left(50 \mathrm{~mJ} / \mathrm{cm}^{2}\right)$, and nuclear extracts were prepared $6 \mathrm{~h}$ after irradiation. (a) Competition of protein binding to T $\beta R \| l$ promoter probe (spanning from -100 to -62$)$ by $50-$ fold molar excess of unlabelled wild-type or mutant NFY probe. (b) Electrophoretic mobility-shift assays (EMSAs) with [ $\left.{ }^{32} \mathrm{P}\right]$ T $\beta R \mathrm{RI}$ promoter probe (spanning from -100 to -62) super shifted with control IgG or anti-NFYA antibody. Closed triangle indicates retarded complex; open triangle indicates super-shifted complex. (c) EMSA with [ $\left.{ }^{32} \mathrm{P}\right]$ NFY consensus probe super shifted with control IgG or anti-NFY-A antibody. Closed triangle indicates retarded complex; open triangle indicates super-shifted complex. Results are representative of three experiments. 

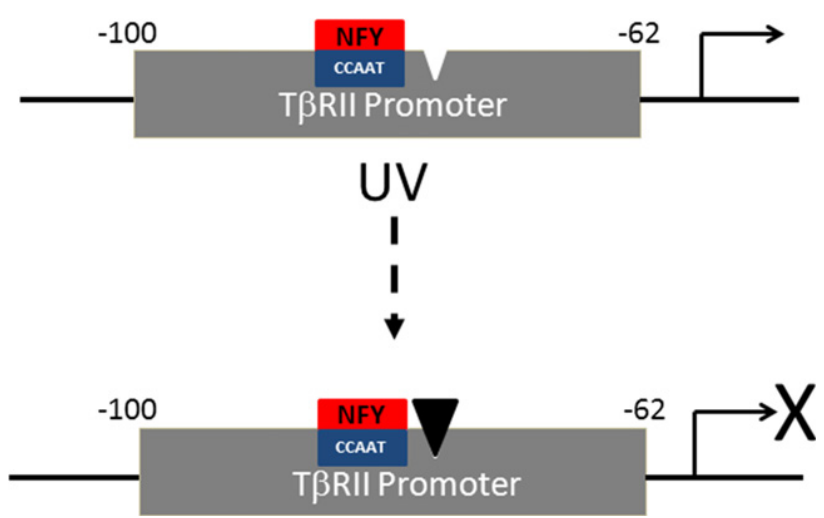

Figure 6. Model of mechanism of inhibition of type II TGF- $\beta$ receptor gene transcription by ultraviolet (UV) irradiation in human skin fibroblasts. An inverted CCAAT box, in the proximal promoter $(-62-100)$ of the TGF- $\beta$ type II receptor (TRRII) gene, is required for transcriptional activity. In adult human skin fibroblasts, nuclear factor $Y$ (NFY) transcription factor complex binds to the inverted CCAAT box. UV irradiation induces binding of an unidentified protein (filled triangle) to the TRRIl proximal promoter. Protein binding antagonizes NFY function, thereby reducing transcription of the T $\beta R \| l$ gene.

In skin dermis, TGF- $\beta$ is a major mediator of fibroblast function and extracellular matrix production. Our previous studies have demonstrated that UV irradiation significantly reduces type I procollagen gene expression, in cultured skin fibroblasts, through impairment of TGF- $\beta$ signalling, largely due to reduction of T $\beta$ RII (11). Restoration of T $\beta$ RII gene expression overcomes the inhibitory effect of UV on type I procollagen production. In addition, knock-down of T $\beta$ RII by siRNA reduces type I procollagen expression, similar to UV irradiation.

In this report, we have investigated regulation of T $\beta$ RII transcription by UV irradiation in human dermal fibroblasts. We identified a 38 bp sequence $(-100 /-62)$, which harbours an inverted CCAAT box, in the proximal T $\beta$ RII promoter that is necessary for promoter activity and confers inhibition by UV irradiation. Reduction in T $\beta$ RII promoter activity by UV irradiation was associated with enhanced protein binding to the 38 bp sequence. NFY binds to the $38 \mathrm{bp}$ promoter region; however, its binding was not altered in response to UV irradiation, implying additional protein(s) are capable of binding to this sequence in response to UV irradiation.
Previous studies have identified several cis-regulatory elements in the T $\beta$ RII gene promoter $(13,20)$. However, the role of these cis-elements in the regulation of T $\beta$ RII gene transcription appears to be complex and cell-type dependent. We found that human skin fibroblasts, transcription of the T $\beta$ RII proximal promoter, is dependent on a $38 \mathrm{bp}$ sequence from -100 to -62 . This sequence also confers regulation by UV irradiation, which inhibits transcription and induces protein binding.

CCAAT box is one the most common cis-elements present in eukaryotic promoters. Various DNA-binding proteins interact with this element, including NFY. NFY is a heteromeric complex composed of three subunits: NFY-A, NFY-B and NFY-C, all required for CCAAT binding. NFY has been shown to exert both positive and negative gene regulation (21-24). We observed that knock-down of NFY-A by siRNA resulted in repression of TßRII promoter activity, indicating NFY function as a positive regulator of T $\beta$ RII gene expression, in cultured human skin fibroblasts. This conclusion is consistent with reports from Park and others, who studied different cell types $(25,26)$.

Nuclear factor Y (NFY) functions via both cooperative and antagonistic interactions with other transcription factors (27-29). Cooperative interactions enhance NFY activity, which could act to repress or stimulate promoter activity, depending on promoter context (3032). Conversely, antagonistic interactions act to diminish NFY function (33-35). Our data demonstrate that NFY binds to an inverted CCAAT box, which is contained within a $38 \mathrm{bp}$ sequence in the T $\beta$ RII proximal promoter. This sequence is required for promoter activity, and repression of activity by UV irradiation is associated with increased protein binding to this sequence. Given that NFY is required for promoter activity, a likely scenario is that increased protein(s)-binding in response to UV irradiation antagonizes NFY function, thereby reducing transcription of the T $\beta$ RII gene (Fig. 6). The identity of the antagonistic protein(s) remains to be determined.

\section{Acknowledgements}

We would like to thank Diane Fiolek for assistance with graphics and administrative assistance. The work was supported, in part, by the National Institute of Health Grant AG019364 to Gary Fisher.

\section{Conflict of interests}

The authors have declared no conflicting interests.

\section{References}

1 Eaglstein W H. Wound healing and aging. Dermatol Clin 1986: 4: 481-484

2 Holt D R, Kirk S J, Regan M C et al. Surgery 1992: 112: 293-297; discussion 297-298.

3 Khorramizadeh M R, Tredget E E, Telasky C et al. Mol Cell Biochem 1999: 194: 99-108.

4 Weinstock M A. J Invest Dermatol 1994: 102 4S-5S.

5 Massague J. Annu Rev Biochem 1998: 67: 753-791.

6 Massague J, Blain S W, Lo R S. Cell 2000: 103 295-309.

7 Massague J, Chen Y G. Genes Dev 2000: 14: 627-644.

8 Fisher G J, Datta S C, Talwar H S et al. Nature 1996: 379: 335-339.

9 Bender K, Blattner C, Knebel A et al. J Photochem Photobiol, B 1997: 37: 1-17.

10 Quan T, He T, Voorhees J J et al. J Biol Chem 2001: 276: 26349-26356.

11 Quan T, He T, Kang S et al. Am J Pathol 2004 165: 741-751.

12 Quan T, He T, Kang $S$ et al. J Invest Dermatol 2002: 118: 402-408.
13 Bae H W, Geiser A G, Kim D H et al. J Bio Chem 1995: 270: 29460-29468.

14 Zhao S, Venkatasubbarao K, Li S et al. Cancer Res 2003: 63: 2624-2630.

15 Quan T, He T, Voorhees J J et al. J Biol Chem 2005: 280: 8079-8085.

16 Benatti P, Dolfini D, Vigano A et al. Nucleic Acids Res 2011: 39: 5356-5368.

17 Quan T, Qin Z, Xu Y et al. J Invest Dermatol 2010: 130: 1697-1706.

18 Inagaki M, Moustakas A, Lin H Y et al. Proc Natl Acad Sci USA 1993: 90: 5359-5363.

19 Sun L, Wu G, Willson J K et al. J Biol Chem 1994: 269: 26449-26455.

20 Yu Y S, Suzuki Y, Yoshitomo $K$ et al. Biochem Biophys Res Commun 1996: 225: 302-306.

21 Boucher P D, Ruch R J, Hines R N. J Biol Chem 1993: 268: 17384-17391.

22 Xu Y, Banville D, Zhao H F et al. Gene 2001 269: 141-153.

23 Kelly D, Kim S J, Rizzino A. J Biol Chem 1998 273: 21115-21124.
24 Bernadt C T, Rizzino A. Mol Reprod Dev 2003: 65: 353-365.

25 Park S H, Lee S R, Kim B C et al. J Biol Chem 2002: 277: 5168-5174.

26 Jin S, Scotto K W. Mol Cell Biol 1998: 18: 4377-4384.

27 Maity S N, de Crombrugghe B. Trends Biochem Sci 1998: 23: 174-178.

28 Mantovani R. Gene 1999: 239: 15-27.

29 Liu J X, Howell S H. Plant Cell 2010: 22: 782-796.

30 Shi $X$, Metges C C, Seyfert H M. BMC Mol Biol 2012: 13: 21.

31 Ito $Y$, Zhang Y, Dangaria S et al. Gene 2011: 473: $92-99$.

32 De Amicis F, Giordano F, Vivacqua $A$ et al. FASEB J 2011: 25: 3695-3707.

33 Barberis A, Superti-Furga G, Busslinger M. Cell 1987: 50: 347-359.

34 Superti-Furga G, Barberis A, Schaffner $G$ et al. EMBO J 1988: 7: 3099-3107.

35 Luo W, Skalnik D G. J Biol Chem 1996: 271: 18203-18210. 